Proceedings of the International Conference on Oxide Materials for Electronic Engineering, May 29-June 2, 2017, Lviv

\title{
Electrocaloric Cooling — A New Application of Relaxor Ferroelectrics
}

\author{
G. SuchaneCK* And G. Gerlach \\ TU Dresden, Solid State Electronics Laboratory, 01062 Dresden, Germany
}

\begin{abstract}
Until now, relaxor ferroelectrics are considered as a class of disordered materials possessing peculiar structures and properties which are not yet generalized into a universal model explaining the significant amount of experimental data available. In this work, we demonstrate that one feature of relaxor ferroelectrics - the extraordinary dielectric response - is well-suited for application in electrocaloric refrigerators. We consider the electrocaloric effect with special attention to relaxor ferroelectrics, the dielectric response in the temperature region of interest, the efficiency and the figure of merit of relaxor ferroelectrics for electrocaloric application.
\end{abstract}

DOI: 10.12693/APhysPolA.133.1003

PACS/topics: 77.70.+a, 84.60.Bk, 77.80.Jk, 77.22.Ch

\section{Introduction}

The electrocaloric (EC) effect is a reversible entropy change in polar dielectrics under an applied electric field. Recently, we have proposed a coefficient of performance of EC elements and a new upper bound of the EC effect which is based on the fact that only a certain energy density might be stored in a dielectric - equivalent to a limit in electrostatic pressure [1]. Correspondingly, a large EC response is expected in materials with large values of thermal diffusivity, dielectric permittivity and its temperature coefficient, as well as high dielectric strength (in order to apply large electric fields), low dielectric and hysteresis losses and a broad temperature region of high dielectric response caused, for instance, by a phase transition. With regard to these requirements, relaxor ferroelectrics (relaxors) appear to be the best choice for EC application. In fact, relaxors such as PVDF-based polymers, lead containing and lead-free perovskite provide sufficient for practical cooling applications EC temperature changes over a broad temperature range [2].

In this work, we consider the EC effect with special attention to relaxors, the dielectric response in the temperature region of interest, the efficiency and the figure of merit of relaxors for EC application.

\section{Electrocaloric effect}

Starting with the Maxwell relation

$$
\left(\frac{\partial S}{\partial E}\right)_{T}=\left(\frac{\partial D}{\partial T}\right)_{E}
$$

where $S$ is the entropy, $E$ - the electric field, $T$ is the temperature, and $D$ - the dielectric displacement

\footnotetext{
* corresponding author; e-mail: Gunnar.Suchaneck@tu-dresden.de
}

$$
D(T, E)=\varepsilon_{0} \varepsilon(E, T) E+P_{r}(T),
$$

with $\varepsilon_{0}$ - the vacuum permittivity, $\varepsilon$ - the relative dielectric permittivity, and $P_{r}$ - the remanent polarization, the EC coefficient for a second-order phase transition is given by [3]:

$$
-\left(\frac{\partial T}{\partial E}\right)_{S}=\frac{T}{c}\left[\varepsilon_{0} E \frac{\partial \varepsilon(E, T)}{\partial T}+\frac{\partial P_{r}(T)}{\partial T}\right],
$$

where $c$ is the volumetric specific heat at constant $E$. The first term in square brackets is determined by the temperature coefficient of dielectric permittivity

$$
T C(E)_{\varepsilon}=\frac{1}{\varepsilon(E, T)} \frac{\partial \varepsilon(E, T)}{\partial T},
$$

while the second term represents the pyroelectric coefficient $p$ under strain-free conditions (crystal volume and shape are fixed). Although the values of $p$ and $\varepsilon$ vary strongly with temperature and from material to material, the ratio $p / \sqrt{\varepsilon}$ is approximately constant for a wide variety of ferroelectrics exhibiting first and second order transitions, as well as displacive and order-disorder types of transitions [4]. Equation (3) then takes the form

$$
-\left(\frac{\partial T}{\partial E}\right)_{S}=\frac{T}{c}\left[\varepsilon \varepsilon_{0} E T C(E)_{\varepsilon}+B \sqrt{\varepsilon}\right],
$$

where $B \approx(3 \pm 1) \times 10^{-5} \mathrm{C} /\left(\mathrm{m}^{2} \mathrm{~K}\right)$ [4]. In Eq. (5), the first term becomes dominant at

$$
\sqrt{\varepsilon} T C_{\varepsilon} E \gg 34 \mathrm{~V} / \mu \mathrm{m} \text {. }
$$

On the other hand, biasing of an EC device is limited by the dielectric strength $E_{\max }$ of the EC material. Taking into account that fact that only a certain energy density might be stored in a dielectric (equivalent to a limit in electrostatic pressure), the value $E_{\max }$ of metal oxide dielectrics is given by an empirical relationship [5]:

$$
E_{\max } \approx 2450 \varepsilon^{-1 / 2} \mathrm{~V} / \mu \mathrm{m} \text {. }
$$

Relaxors, in particular $\mathrm{BaZr}_{0.25} \mathrm{Ti}_{0.75} \mathrm{O}_{3}$ (BZT25) [6, 7], $\mathrm{BaZr}_{0.3} \mathrm{Ti}_{0.7} \mathrm{O}_{3} \quad(\mathrm{BZT} 30) \quad[8], \quad \mathrm{BaZr}_{0.35} \mathrm{Ti}_{0.65} \mathrm{TiO}_{3}$

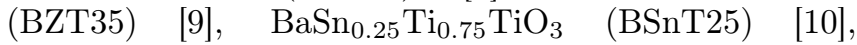
$\mathrm{Ba}_{0.20} \mathrm{~Pb}_{0.80} \mathrm{ZrO}_{3}$ (BPZ80) [11], and solid solutions of 
0.9 $\mathrm{PbMg}_{1 / 3} \mathrm{Nb}_{2 / 3} \mathrm{O}_{3}-0.1 \mathrm{PbTiO}_{3} \quad(90 \mathrm{PMN}-10 \mathrm{PT})$ [12], possess values of $T C_{\varepsilon}$ as high as a few $10^{-2} \mathrm{~K}^{-1}$. Here, in the limit of $E=E_{\max }$, Eq. (6) is fulfilled for any dielectric permittivity. For a dominating first term of Eq. (5), the electrocaloric temperature change $\Delta T_{E C}$ is given by

$$
\Delta T_{E C} \approx-\frac{\varepsilon_{0} T}{c} \int_{E_{1}}^{E_{2}} \frac{\partial \varepsilon(T, E)}{\partial T} \mathrm{~d} E .
$$

\section{Relaxor ferroelectrics}

Relaxors are a class of disordered crystals possessing peculiar structure and properties [13]. They exhibit frequency dispersion, i.e., the temperature $T_{m}$ of the dielectric constant maximum increases with increase of frequency. In relaxors, polar entities, e.g. polar nanoregions (PNR), appear at temperatures much higher than the Curie point, but below the so-called Burns temperature. Dynamic PNRs are not subjected to dielectric losses characteristic of polar domains. The huge dielectric response and the existence of a maximum of $\varepsilon(T)$ is attributed to the peculiarities of the PNR behavior. Above the temperature of maximum dielectric permittivity up to a temperature $T^{*}$ where static PNRs disappear [14], relaxors provide a large and reversible polarization change due to contributions to polarization not present in ordinary ferroelectrics [13]. This is just required for a large EC response. In this temperature region, the dielectric response is dominated by the static dielectric susceptibility which is given by the empirical formula [13]

$$
\chi_{s}(T)=\varepsilon_{s}(T)-1=\frac{\chi_{A}}{1+\left(\frac{T-T_{A}}{2 \Omega}\right)^{2}},
$$

where $\chi_{A}, T_{A}$ and $\Omega$ are frequency independent fitting parameters and the subscript $s$ denotes static. Here, the value of $\Omega$ characterizes the width of the permittivity peak. The first and second temperature derivatives of the dielectric permittivity are then easily calculated. In order to fulfill the condition that heat rejected to the sink is larger than heat absorbed from the load $\left(\partial^{2} \varepsilon / \partial T^{2}\right.$ $>0)$ [15], relaxors must be driven at a certain temperature above the temperature of maximum dielectric permittivity

$$
T_{\text {min }}-T_{A}=\frac{2 \Omega}{\sqrt{3}} .
$$

This results in an employable temperature range of $T_{\text {min }}$ $<T<T^{*}$. This temperature range can be adapted to the desired one by selecting a proper relaxor composition, e.g., by varying the PT fraction in PMN-PT solid solutions [16] or the Zr-fraction in BZT [17].

\section{Electrocaloric relaxor materials}

Refrigerant materials are evaluated by a materials criterion which characterizes the efficiency of the physical cooling process and which, therefore, is independent of the performance of different thermodynamic cycles. For EC cooling it is given by [3]:

$$
\Phi_{\text {mat }}=1-\frac{\varepsilon \varepsilon_{0} E^{2} \tan \delta}{c \Delta T_{E C}},
$$

where $\varepsilon \varepsilon_{0} E^{2} \tan \delta$ is the non-recoverable electrical loss with $\tan \delta$ - the loss tangent, and $c \Delta T_{E C}$ the transferred from the load to the heat sink within one refrigeration cycle. The EC temperature change was calculated by means of Eq. (8) for a given field change $\Delta E=E_{2}-E_{1}$. Note that for relaxor ferroelectrics $\left\langle P_{r}\right\rangle=0$.

Table I compares the materials efficiency $\Phi_{\text {mat }}$ of promising relaxor ferroelectric EC refrigerants and the corresponding figure of merit (FOM) recently derived in [3]:

$$
F O M=\frac{\kappa c \Delta T_{E C}^{2} \delta T}{\varepsilon \varepsilon_{0} \tan \delta} \frac{1}{T E^{2} d^{2}},
$$

where $\kappa$ is the thermal conductivity, $\delta T$ - the empirical full width at half maximum of the $\Delta S$ vs. $T$ curve, and $d$ - the thickness of the EC layer. This FOM consists of a term describing the properties of the EC material and a term of operational parameters applied to the material. For comparison, a thickness of the EC layer of $100 \mu \mathrm{m}$ was chosen. For sake of simplicity, the field dependences of the specific heat $c$ and thermal conductivity $\kappa$ were neglected. To account for the field dependence of the dielectric permittivity, an average value of $\varepsilon$ should be calculated for the given electric field region. If higher order terms of the Landau-Ginsburg-Devonshire thermodynamic potential are neglected, the field dependence of dielectric permittivity can be written in an explicit form $[18,19]$ :

$$
\varepsilon(E) \approx \frac{\varepsilon_{0} \varepsilon_{r}(0)}{\left(1+a E^{2}\right)^{1 / 3}}=\varepsilon_{1}-\varepsilon_{2} E^{2}+\varepsilon_{3} E^{4}-\ldots
$$

where $a=3 b\left[\varepsilon_{0} \varepsilon_{r}(0)\right]^{3}, b$ is a temperature-independent quartic coefficient of the Landau-Ginsburg-Devonshire equation, $\varepsilon_{r}(0)$ is the relative dielectric permittivity at zero field, $\varepsilon(0)=\varepsilon_{0} \varepsilon_{r}(0)$, and $\varepsilon_{1}=\varepsilon(0), \varepsilon_{2}=(1 / 3) a \varepsilon(0)$, $\varepsilon_{3}=(2 / 9) a^{2} \varepsilon(0)$, etc. The parameter $\varepsilon_{1}$ is the linear dielectric permittivity. The higher order terms $\varepsilon_{2}, \varepsilon_{3}$, etc. are nonlinear dielectric permittivities which are all temperature dependent. The series expansion of Eq. (13) is valid up to electric field in the order of $1 \mathrm{MV} / \mathrm{m}$. At larger fields $E>2 a^{-1 / 2}$, averaging over $E^{2}$ yields

$$
\langle\varepsilon\rangle=\frac{3 \varepsilon(0)}{2 a^{1 / 3} E^{2 / 3}} .
$$

Polymer relaxor ferroelectrics are fabricated either by high-energy irradiation of poly(vinylidene fluoridetrifluoroethylene) (P(VDF-TrFE) ) copolymer or by copolymerization of $\mathrm{P}(\mathrm{VDF}-\mathrm{TrFE})$ with a bulky monomer such as CFE (chlorofluoroethylene) disrupting the long-range ferroelectric order [20]. PVDF-based relaxors possess a weak dependence of dielectric permittivity on electric field, i.e., $a \approx 0$. Values of $a$ for other compounds were estimated by fitting the values of $\varepsilon(E)$ for BZT25 and BZT30 [17], 0.7PMN-0.3PT [21], 0.91PMN-0.09PT and PMN [19], and PLZT8/85/15 [22] to Eq. (13). Since Eq. (13) is valid for small enough polarization, only values $\varepsilon(E)$ at fields less than $1 \mathrm{~V} / \mu \mathrm{m}$ were taken into account. 
TABLE I

Material characteristics, the materials efficiency $\Phi_{m a t}$ and the figure of merit FOM selected EC refrigerants.

\begin{tabular}{c|c|c|c|c|c|c|c|c|c|c}
\hline \hline Refrigerant & $\begin{array}{c}T \\
{[\mathrm{~K}]}\end{array}$ & $\begin{array}{c}\Delta T_{E C} \\
{[\mathrm{~K}]}\end{array}$ & $\begin{array}{c}\Delta E \\
{[\mathrm{~V} / \mu \mathrm{m}]}\end{array}$ & $\begin{array}{c}c \\
{\left[\mathrm{MJ} / \mathrm{m}^{3} \mathrm{~K}\right]}\end{array}$ & $\varepsilon$ & $\begin{array}{c}\kappa \\
{[\mathrm{W} /(\mathrm{m} \mathrm{K})]}\end{array}$ & $\begin{array}{c}\delta T \\
{[\mathrm{~K}]}\end{array}$ & $\tan \delta$ & $\Phi_{\text {mat }}$ & $\begin{array}{c}\text { FOM } \\
{\left[\mathrm{mW} / \mathrm{cm}^{3}\right]}\end{array}$ \\
\hline $\begin{array}{c}\mathrm{P}(\mathrm{VDF})-\text { based } \\
\text { polymers }\end{array}$ & 305 & 20 & 200 & 2.7 & 60 & 0.2 & 50 & 0.15 & 0.941 & 1.111 \\
\hline BaZr $_{0.2} \mathrm{Ti}_{0.8} \mathrm{O}_{3}$ & 310 & 4.5 & 15 & 3.4 & 800 & 2.6 & 30 & 0.05 & 0.994 & 21.739 \\
\hline $0.7 \mathrm{PMN}-0.3 \mathrm{PT}$ & 420 & 2.5 & 10 & 2.8 & 6000 & 1.5 & 100 & 0.08 & 0.949 & 1.765 \\
\hline $0.9 \mathrm{PMN}-0.1 \mathrm{PT}$ & 350 & 5 & 90 & 3 & 1250 & 1.3 & 100 & 0.1 & 0.986 & 0.311 \\
\hline $\mathrm{PMN}$ & 340 & 2.5 & 9 & 2.6 & 2000 & 1.3 & 100 & 0.08 & 0.982 & 5.415 \\
\hline $\mathrm{PLZT8/65/35}$ & 385 & 2.5 & 10 & 3 & 5000 & 2.3 & 80 & 0.1 & 0.991 & 1.320 \\
\hline PLZT8/65/35 & 318 & 40 & 120 & 3 & 1000 & 2.3 & 80 & 0.07 & 0.963 & 20.295
\end{tabular}

The obtained values of $\Phi_{\text {mat }}$ exceed significantly the ones known for the Brayton combustion engines (0.60.8). On the other hand, the actual FOM will depend on the field dependence of $\varepsilon$ and on the fraction of $\delta T$ implemented by the actual temperature span of the refrigerator. By multiplying FOM with the thickness of the EC element, it gives an estimate of the cooling power per area achievable. Since $c$ is close to the Dulong-Petit limit and the ratio $\kappa / c$ determined by the phonon mean free path (a few unit cell dimensions in perovskites) is nearly constant [2], the largest impact on the FOM (assuming a given temperature span) arises from the EC temperature change $\Delta T_{E C}$ which on its part is determined by the temperature coefficient of the dielectric permittivity $\varepsilon$ (cf. Eqs. (5) and (11)). This is the point where the peculiar properties of relaxors come into play.

\section{Conclusions}

Due to their extraordinary dielectric response, relaxor ferroelectrics are promising for application in electrocaloric refrigerators. The operational temperature of such devices may be tuned by selecting proper compositions of perovskite solid solutions possessing relaxor properties.

\section{References}

[1] G. Suchaneck, G. Gerlach, Mater. Today Proc. 3, 622 (2016).

[2] G. Suchaneck, in: Proc. 2016 Joint IEEE Int. Symp. on the Applications of Ferroelectrics, European Conf. on Application of Polar Dielectrics, and Piezoelectric Force Microscopy Workshop (ISAF/ECAPD/PFM), Darmstadt (Germany), 2016.

[3] G. Suchaneck, O. Pakhomov, G. Gerlach, in: Refrigeration, Ed. Orhan Ekren, Intech, Rijeka 2017, pp. 19-43.
[4] S.T. Liu, J.D. Zook, D. Long, Ferroelectrics 9, 39 (1975).

[5] J. McPherson, J.-Y. Kim, A. Shanware, H. Mogul, Appl. Phys. Lett. 82, 2121 (2003).

[6] R.C. Kell, N.J. Hellicar, Acustica 6, 235 (1956).

[7] D. Hennings, A. Schnell, G. Simon, J. Am. Ceram. Soc. 65, 539 (1982).

[8] Zhi Yu, Chen Ang, Ruyan Guo, A.S. Bhalla, J. Appl. Phys. 92, 2655 (2002).

[9] W. Kleemann, S. Miga, J. Dec, J. Zhai, Appl. Phys. Lett. 102, 232907 (2013).

[10] C. Lei, A.A. Bokov, Z.-G. Ye, J. Appl. Phys. 101, 084105 (2007).

[11] S. Roberts, J. Am. Ceram. Soc. 33, 63 (1950).

[12] S. Hirose, T. Usui, S. Crossley, B. Nair, A. Ando, X. Moya, N.D. Mathur, APL Mater. 4, 064105 (2016).

[13] A. Bokov, Z.-G. Ye, J. Adv. Diel. 2, 1241010 (2012).

[14] W. Dmowski, S.B. Vakhrushev, I.-K. Jeong, M.P. Hehlen, F. Trouw, T. Egami, Phys. Rev. Lett. 100, 137602 (2008).

[15] G. Suchaneck, G. Gerlach, Phase Transit. 88, 333 (2015).

[16] J. Peräntie, H.N. Tailor, J. Hagberg, H. Jantunen, Z.-G. Ye, J. Appl. Phys. 114, 174105 (2013).

[17] X.G. Tang, K.-H. Chew, H.L.W. Chan, Acta Mater. $\mathbf{5 2}, 5177$ (2004).

[18] K.M. Johnson, J. Appl. Phys. 33, 2826 (1962).

[19] Biaolin Peng, Huiqing Fan, Qi Zhang, J. Am. Ceram. Soc. 95, 1651 (2012).

[20] B. Neese, Baojin Chu, Sheng-Guo Lu, Yong Wang, E. Furman, Q.M. Zhang, Science 321, 821 (2008).

[21] R. Herdier, M. Detalle, D. Jenkins, C. Soyer, D. Remiens, Sens. Actuat. A 148, 122 (2008).

[22] Ye Zhao, Xihong Hao, Qi Zhang, ACS Appl. Mater. Interfaces 6, 11633 (2014). 